\title{
Treatment of attention deficit hyperactivity disorder insomnia with blue wavelength light-blocking glasses
}

This article was published in the following Dove Press journal:

ChronoPhysiology and Therapy

4 January 2013

Number of times this article has been viewed

\section{Rachel E Fargason \\ Taylor Preston \\ Emily Hammond \\ Roberta May \\ Karen L Gamble}

Department of Psychiatry and

Behavioral Neurobiology, University

of Alabama at Birmingham School

of Medicine, Birmingham, AL, USA
Correspondence: Rachel Fargason 3rd Floor Callahan Eye Foundation Hospital, 1720 University Boulevard, Birmingham, AL 35294, USA

Tel + I 205934430 I

Fax + I 2059759600

Email Rfargason@uab.edu
Background: The aim of this study was to examine a nonmedical treatment alternative to medication in attention deficit hyperactivity disorder (ADHD) insomnia, in which blue wavelength light-blocking glasses are worn during the evening hours to counteract the phase-delaying effect of light. Outcome measures included sleep quality and midsleep time. The capacity of ADHD subjects to comply with treatment using the glasses was assessed.

Methods: Daily bedtime, wake-up time, and compliance diaries were used to assess sleep quality and timing during a baseline observation week and a 2-week intervention period. The Pittsburgh Sleep Quality Index (PSQI) was administered following baseline and intervention. The intervention protocol consisted of use of blue wavelength-blocking glasses and a moderate lighting environment during evening hours.

Results: Partial and variable compliance were noted, with only 14 of 22 subjects completing the study due to nonadherence with wearing the glasses and diary completion. Despite the minimum 3-hour recommendation, glasses were worn, on average, for 2.4 hours daily. Lighting was reduced for only $58.7 \%$ of the evening. Compared with baseline, the intervention resulted in significant improvement in global PSQI scores, PSQI subcomponent scores, and sleep diary measures of morning refreshment after sleep $(P=0.037)$ and night-time awakenings $(P=0.015)$. Global PSQI scores fell from 11.15 to 4.54, dropping below the cut-off score of 5 for clinical insomnia. The more phase-delayed subjects, ie, those with an initial midsleep time after 4:15 am, trended towards an earlier midsleep time by 43.2 minutes following the intervention $(P=0.073)$. Participants reported less anxiety following the intervention $(P=0.048)$.

Conclusions: Despite only partial compliance with intervention instructions, subjects completing the study showed subjectively reduced anxiety and improved sleep quality on multiple measures. The more sleep-delayed subjects trended toward an earlier sleep period following use of the glasses. Blue-blocking glasses are a potential insomnia treatment for more compliant subjects with ADHD insomnia, especially those with prominent sleep delay. Larger studies of blue light-blocking glasses in more phase-delayed groups could reveal significant advances in chronotherapeutics.

Keywords: insomnia, attention deficit hyperactivity disorder, circadian rhythm disorders, chronobiological treatment

\section{Introduction}

Attention deficit hyperactivity disorder (ADHD) insomnia is a prevalent problem. An estimated $4.4 \%$ of the adult population suffers from ADHD, with over $70 \%$ of these individuals experiencing insomnia., ${ }^{1,2}$ Dim-light melatonin onset studies and clinical studies using DSM-IV-TR (Diagnostic and Statistical Manual of Mental Disorders, Fourth Edition, Text Revision) criteria have demonstrated that two-thirds of subjects 
with ADHD insomnia have circadian rhythm sleep disorder, delayed sleep phase type, ${ }^{3,4}$ and one-third have primary insomnia without evidence of circadian delay. ${ }^{4}$ Even groups of ADHD subjects who do not report sleep problems often manifest clinically significant insomnia., ${ }^{3,5}$ Recent studies show a link between ADHD and genes involved in the circadian timekeeping system. ${ }^{6,7}$ A polymorphism at the 3 -untranslated region of the circadian locomotor output cycles kaput (CLOCK) gene is associated with adult ADHD. ${ }^{6}$ It is unknown whether a common genetic link underlies both the ADHD and the circadian disorder, or if the sleep disorder is simply a modifier of ADHD symptomatology. Intrinsically photoreceptive melanopsin-containing retinal ganglion cells are primarily responsible for transmitting the presence of short-wavelength light to the primary circadian pacemaker in the suprachiasmatic nucleus, which in turn suppresses the hormone melatonin. ${ }^{8}$ ADHD subjects have suppressed, shortened, and delayed evening melatonin secretion, which affects both melatonin-related hypnotic and circadian phase resetting functions, as well as bodily functions such as blood pressure, thermoregulation, and glucose regulation. ${ }^{3,9}$ Exogenous melatonin is commonly used to treat insomnia in ADHD, but study results for efficacy are conflicting, ${ }^{10}$ as a result of the varying potency of a substance not approved by the US Food and Drug Administration and the individual variability in timing and dosing needs of melatonergic agents. ${ }^{11,12}$ Another study of chronobiological treatments for ADHD demonstrated that advancing the circadian phase in ADHD subjects by use of morning bright light therapy is strongly correlated with improvement in core ADHD symptoms. ${ }^{13}$ Despite the positive results of that study, it has not been replicated with objective measures of circadian phase. Chronobiological treatments, such as bright light therapy, have become the standard of care for some psychiatric conditions, such as seasonal affective disorder, ${ }^{14}$ and are gaining momentum with the use of total sleep deprivation to advance circadian phase in manic bipolar subjects in order to stabilize mood. ${ }^{15}$ Despite recent advances, many potential uses of chronobiological treatments for psychiatric disorders with circadian disruption, including ADHD, are as yet unexplored.

Chronic exposure to electrical lighting in the evening further disrupts melatonin signaling in both ADHD and control subjects, suppressing and delaying the normal nocturnal rise in melatonin to a profound degree, and shortening melatonin duration and the body's internal representation of night. ${ }^{16}$ Melatonin suppression, with associated insomnia, is particularly sensitive to light in the blue wavelength (about $460 \mathrm{~nm}$ ) emitted from cell phones, computers, and television. ${ }^{17}$ Self-medication for insomnia is common, and medications prescribed for insomnia carry the risk of addiction, accidents, cognitive impairment, and medical side effects. ${ }^{18,19}$ Nonpharmacological chronobiological treatments are low risk, and specifically address the circadian deficit in ADHD insomnia. Because neural pathways from the retina to the primary circadian pacemaker in the suprachiasmatic nucleus respond only to shorter wavelength light of $<550 \mathrm{~nm}$, physiological darkness can be produced by use of blue wavelength-blocking glasses with the advantage of continued evening functioning. Blue light-blocking glasses have been shown to preserve normal evening night-time melatonin production in subjects exposed to light. ${ }^{20,21}$ One randomized study of subjects with insomnia demonstrated improved sleep quality and mood in an intervention group wearing blue wavelength-blocking glasses, compared with a placebo group. ${ }^{22}$ Daytime use of the glasses in permanent night-shift workers resulted in longer sleep, better daytime sleep efficiency, and less sleep fragmentation. ${ }^{23}$ Analogously, darkness treatment improved sleep and mood in subjects with bipolar disorder. ${ }^{24,25}$

This is the first study to examine the chronobiological treatment of blue wavelength-blocking glasses in adults with ADHD insomnia. It explores the primary hypothesis that wearing glasses that filter blue wavelength light from overhead lights and electronic appliances will counteract the deleterious effects of evening light on sleep, thereby advancing sleep phase. In addition, we hypothesize that use of these glasses will improve sleep quality measures, such as sleep efficiency, total sleep time, and sleep fragmentation in subjects with ADHD insomnia, providing a potential nonmedical treatment alternative to hypnotic medication. Finally, we explore the feasibility of this regular and sustained intervention for sleep behavior in the adult ADHD population, which is a population at risk for poor compliance.

\section{Materials and methods}

Subjects aged 19 years and older attending the Adult ADHD Clinic, Department of Psychiatry, University of Alabama at Birmingham School of Medicine (UAB) were recruited for this 3-week study extending from fall 2011 to spring 2012. The UAB institutional review board approved the study and all participants signed an informed consent form prior to participation. Participants were informed that the study explored a treatment for delayed sleep rhythms; all subjects agreed to treatment in anticipation of this specific benefit. 
Inclusion criteria included a DSM-IV-TR diagnosis of both ADHD and circadian rhythm sleep disorder, delayed sleep phase type, by physician-administered semistructured interview and the ADHD-Rating Scale-IV validated diagnostic scale. Exclusion criteria included shift-work, use of sleep medication, active or severe psychiatric disorders, substance abuse or dependence, sleep disorders other than insomnia, chronic pain, and active or severe medical problems. In determining not to have an age cut-off for this pilot study, we considered typical age-related alterations in sleep patterns. However, all study subjects, including older ones, reported delayed sleep patterns significant enough to cause daytime dysfunction, and hence met DSM-IV-TR criteria for circadian rhythm sleep disorder, delayed sleep phase type, which is a diagnosis made by clinical history. These subjects resembled younger ADHD subjects by history much more than their same-age peers, who in the absence of ADHD, typically have more advanced sleep cycles. Also, while older subjects demonstrate diminished arousal responses to bright light exposure compared with younger subjects, phase-delaying responses to light are undiminished. ${ }^{26,27}$ Because the primary outcome measure for this study was phase change from baseline resulting from intervention using the experimental glasses, absolute baselines were less important. Medication risks make subjects of advanced age most needful of nonpharmacological treatments for delayed circadian rhythm sleep disorder.

\section{Procedure}

The first week was the baseline assessment period. Assessment measures were collected while the subjects engaged in their usual evening activities. Following a reminder call, the 2-week intervention period began. Participants were instructed to wear a set of amber glasses that blocked wavelengths of light from $530 \mathrm{~nm}$ and below (blue and violet wavelengths of light) from sundown until bedtime every evening. Subjects wearing corrective lenses wore amber glasses designed to fit over their own pair of glasses. LowBlueLights.com (Photonic Developments LLC and the Lighting Innovations Institute) manufactured the glasses and generously provided them for this project. One recent study demonstrated that ordinary yellow-tinted "placebo" glasses had partial blue wavelength-blocking effects. ${ }^{22}$ In the absence of a viable placebo option, we elected for an open trial format using the baseline period for comparison. Participants were instructed to wear the glasses every evening (beginning at sundown) for a minimum of 3 hours prior to bedtime until turning the lights out to sleep. Subjects were instructed to create a moderate light environment by using only floor and table lamps and avoiding overhead lights during the evening. Reduction of the typical evening bright light environment to a moderate light environment was incorporated into the intervention protocol to reduce unfiltered light entering the retina from the periphery of the glasses, which might otherwise have confounded our study results. Reducing bright light to moderate levels alone could not significantly alter the study results, because even moderate indoor lighting environments with the 200 lux intensity required to concentrate on detail inhibit nocturnal melatonin onset and delay sleep phase. ${ }^{28}$ True dim-light conditions of $<60$ lux do not suppress melatonin onset but are impractical, and a single candle is $20-30$ lux..$^{28}$ If participants awoke during the night, they were instructed to put the glasses on before turning on a lamp. Subjects otherwise engaged in their usual evening activities and routine. All subjects but one took optimized doses of ADHD medications and were instructed to continue taking their regular regimen during the study. Subjects were instructed to minimize caffeine, nicotine, and alcohol, avoiding caffeine after lunch and alcohol and smoking after dinner. Instructions were provided verbally and in writing. Compliance with instructions was assessed daily.

\section{Assessment measures}

Standard bedtime and wake-up diaries were assessed daily during the first baseline week to capture information regarding the participant's subjective emotional state, bedtime, sleep latency, nocturnal wakening, time spent awake in the night, wake time, and feelings of alertness and refreshment upon waking. During weeks 2 and 3, ie, the intervention period, participants completed sleep diaries with additional questions designed to measure compliance with protocol instructions.

\section{Pittsburgh Sleep Quality Index}

The Pittsburgh Sleep Quality Index (PSQI) was measured at the end of the baseline assessment week and again after the intervention. The PSQI is a validated self-rating instrument assessing aspects of sleep quality. ${ }^{29}$ Field-testing of the PSQI shows high sensitivity and specificity in differentiating persons with insomnia from unaffected controls when the score is $>5 .{ }^{30}$ The PSQI is considered appropriate in identifying "new-onset" insomnia in the clinical setting. ${ }^{30,31}$

\section{Compliance measures}

During the intervention period, nightly diary questions recorded the precise times the glasses were worn, 
the relationship between removal of glasses to lights-out time, and the percent of evening time the lights were kept dimmed.

\section{Statistical analysis}

Advances in sleep rhythms were measured by midsleep time, ie, the time point midway between sleep time and waking time. Midsleep time is a reliable clinical measure of circadian phase in the absence of other physiological measures. ${ }^{32}$ Sleep diaries and the PSQI were compared for baseline versus intervention conditions by two-tailed paired $t$-tests. A two-way analysis of variance was used to compare midsleep times following intervention in phase subgroups of subjects.

\section{Results}

\section{Participant demographics}

Twenty-two subjects entered the study, with 14 subjects completing it. Six subjects never began the study due to illness, sudden travel, or irregular schedules, and two motivated subjects were (with some embarrassment) unable to complete it. All early terminations involved poor motivation to complete the diaries or forgetting to wear the glasses. Subjects were aged $21-76$ years, with a mean age of 43.9 years. The study population was $43 \%$ male and $57 \%$ female, $64.2 \%$ were married or cohabitating, $43 \%$ had post-graduate degrees, and all subjects had some post high school training. Only $7.1 \%$ of subjects were unemployed.

\section{Participant compliance}

During the intervention period, the glasses were worn for, on average, 2.4 hours daily (instructions were for 3 hours minimum). Glasses were put on at a mean decimal time of 20.7 (about 8:45 pm) and taken off at 23.20 (about 11:10 pm). A high variability in compliance was noted between subjects, with some starting as early as 18.95 (before 7:00 pm) and others as late as 23.23 (about 11.15 pm). Subjects had been instructed to wear the glasses until the lights were turned off at bedtime. Glasses were removed on average 0.52 hours (slightly over 30 minutes) before bed, but this time interval varied from 0.47 hours after turning off lights to 3.15 hours before bedtime. The average percentage of evening time subjects kept overhead lights off after sundown, as instructed, was $58.7 \%$, but ranged from $7 \%$ to $91 \%$ of evening hours. Outside light exposure varied from 11 minutes to 3.83 hours and averaged 1.51 hours. One subject reported a mild intermittent headache, possibly related to wearing the glasses and another subject reported pain on the bridge of the nose from the glasses.

\section{PSQI sleep quality}

We next examined whether sleep quality improved with the intervention (Table 1). Significant improvement was noted on global PSQI scores as well as nearly every subcomponent following the intervention, with the exception of "sleep medications," which were not permitted during the study. Global PSQI scores fell from a mean of $11.15 \pm 3.50$ to $4.54 \pm 3.15$ following the intervention. Scores below the cut-off of 5 are considered to be within the normal range. Figure 1 depicts the individual global PSQI scores both before and after intervention using the experimental glasses.

\section{Sleep diary variables}

In order to determine the effect of intervention on daily self-reported sleep measures, paired-samples $t$-tests were run for each subject before and after intervention using the experimental glasses. Statistically significant differences were noted on sleep diary items for the number of nighttime awakenings and "feeling refreshed on awakening". Feeling refreshed after sleep was significantly improved after the intervention compared with baseline $[t(13)=2.329$, $P=0.037]$. Night-time awakenings were significantly reduced following the intervention compared with baseline $[t(13)=2.805, P=0.015]$. To compare the consistency of the sleep diary with the PSQI parameters, we compared the reported sleep latency values. These two measures were significantly correlated with each other [Pearson's correlation, $R(14)=0.585, P=0.028]$.

\section{Midsleep time}

We hypothesized that blocking evening blue light exposure may not advance circadian phase but may prevent further phase delay. Therefore, we sought to examine whether

Table I Pittsburgh Sleep Quality Index results

\begin{tabular}{|c|c|c|c|c|c|c|}
\hline & \multicolumn{3}{|c|}{ Pair differences } & \multirow[t]{2}{*}{$\boldsymbol{t}$} & \multirow[t]{2}{*}{ df } & \multirow{2}{*}{$\begin{array}{l}\text { Significance } \\
\text { (two-tailed) }\end{array}$} \\
\hline & Mean & SD & SEM & & & \\
\hline Sleep quality & 0.62 & 0.65 & 0.18 & 3.4 & 12 & 0.005 \\
\hline Sleep latency & 0.69 & 0.75 & 0.21 & 3.3 & 12 & 0.006 \\
\hline Total sleep time & 1.46 & 1.13 & 0.31 & 4.7 & 12 & 0.001 \\
\hline Sleep efficiency & 1.23 & 1.01 & 0.28 & 4.4 & 12 & 0.001 \\
\hline Sleep disturbance & 1.46 & 0.52 & 0.14 & 10.2 & 12 & 0.000 \\
\hline Sleep medications & 0.39 & 0.77 & 0.21 & 1.8 & 12 & 0.096 \\
\hline Daytime dysfunction & 0.77 & 0.73 & 0.20 & 2.8 & 12 & 0.002 \\
\hline Global PSQI & 6.62 & 2.4 & 0.68 & 9.8 & 12 & 0.00 \\
\hline
\end{tabular}

Notes: Table depicts mean decrease in scores (lower score means less impaired) on each component of the PSQI and the Global PSQI. Statistical significance was noted on each measurable component.

Abbreviations: SD, standard deviation; SEM, standard error of the mean; PSQI, Pittsburgh Sleep Quality Index. 


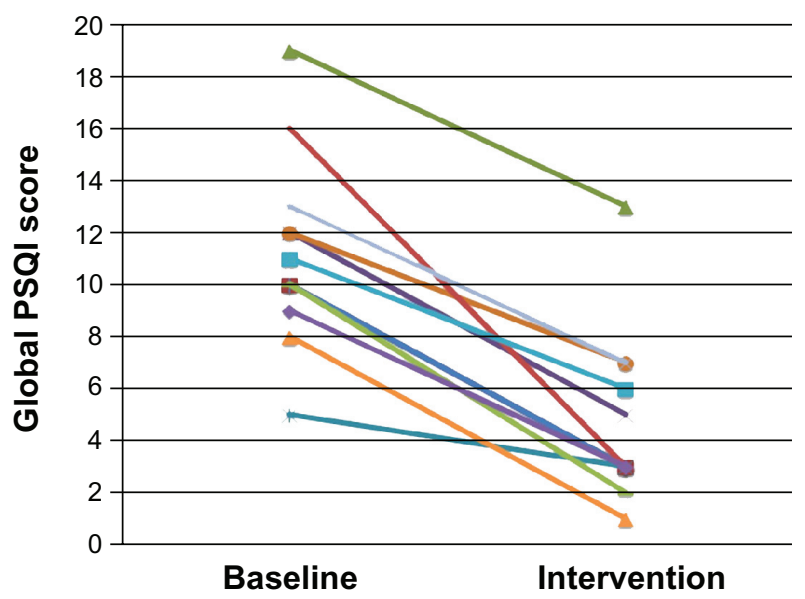

Figure I Individual PSQI global scores. Global PSQI scores are shown for each subject before and after the glasses intervention.

Note: Subjects' mean scores dropped from II.I5 to 4.54 , below the clinically impaired score of 5 and into the normal range.

Abbreviation: PSQI, Pittsburgh Sleep Quality Index.

wearing the glasses was more effective for subjects who were more delayed to start with. Midsleep time for the baseline circadian phase was used to divide the cohort into two groups using the 50th percentile, with 4.25 decimal time (4:15 am) the resulting cut-off, leaving an earlier group with midsleep time before 4.25 and a more delayed group with midsleep time 4.25 and later. A two-way repeatedmeasures analysis of variance revealed no main effect of the intervention $[F(1,12)=2.77, P=0.122]$, although there was a strong trend toward a phase type $\mathrm{X}$ intervention interaction $[F(1,12)=3.85, P=0.073]$, such that the glasses condition tended to shift midsleep time earlier in the more delayed group (Figure 2). The more delayed group shifted to a mean of 0.72 hours (43.2 minutes) earlier following

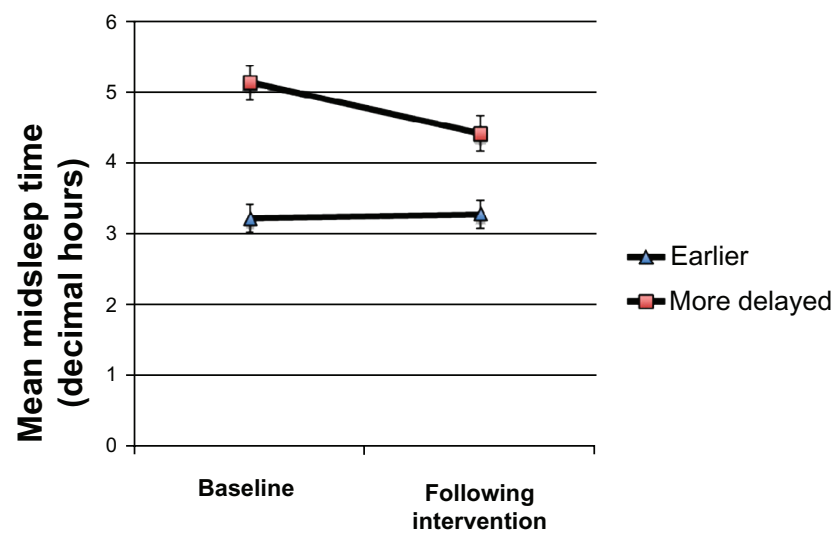

Figure 2 Midsleep time.

Notes: Mean midsleep time is compared before and after intervention with glasses for the earlier, $\leq 4.25$ decimal time, and more delayed groups, $4.25+$ decimal time. $\mathrm{n}=7$ for each group. The earlier group shows little change following intervention but the more delayed group demonstrates a positive trend, with an average 43 minutes advance in midsleep time following the intervention $(P=0.073)$. the intervention. No change in circadian phase was seen in the earlier group following intervention.

\section{Sleep diary mood scores}

Although clinical levels of anxiety and depression were exclusion criteria for the study, we compared the mood ratings for the sleep diary before and after the intervention using paired $t$-tests. Participants indicated being significantly less worried (the cognitive component of anxiety), following the intervention [mean \pm standard error of the mean: baseline $3.27 \pm 0.17$; intervention $3.48 \pm 0.15, t(13)=-2.178$, $P=0.048]$. Other measures, such as jitteriness, moodiness, sadness, tension, irritability, and poor concentration, were unchanged $(P>0.05)$.

\section{Discussion}

Use of the blue wavelength light-blocking glasses did not significantly advance the circadian phase outcome measure of midsleep time as expected. However, the seven subjects who had more delayed sleep times advanced their sleep time by an average of 43 minutes following the intervention, a positive trend that with a larger sample size could reach significance. In large pharmaceutical trials, improvements in sleep latency of 15-29 minutes were clinically significant. ${ }^{33,34}$ By contrast, robust improvements occurred in sleep quality as measured by global PSQI scores, despite the small sample size, attesting to the size of the measured effect. Post-intervention scores decreased to less than half of the baseline scores. Moreover, the notable improvement in the PSQI subcomponents confirmed the significant sleep diary findings of increased morning refreshment and decreased night-time awakenings. The correlation between PSQI and sleep diary findings strengthens the consistency of these findings. Of interest, while all sleep quality subcomponents measured were significantly improved, the subjects' chief complaints were not of sleep quality problems, but circadian phase delays. Several subjects verbally reported "no improvement" from wearing the glasses in spite of positive improvement on the research measures.

A recent study showed that abnormal sleep quality, and in particular daytime sleepiness, was present even in ADHD subjects who did not report insomnia or a circadian delay complaint (as measured by the detailed PSQI scale). ${ }^{5}$ Likewise, subjects in this study were not aware of all aspects of their insomnia until specifically questioned by the detailed PSQI. The robust improvement in these relatively unrecognized symptoms with the glasses intervention suggests that interventions that address circadian components 
of insomnia may also improve sleep quality issues assessed by the PSQI, such as sleep latency, sleep efficiency, total sleep time, daytime sleepiness, and night-time arousals.

One polysomnography study demonstrated that evening light exposure had a seeming alerting effect persisting into the sleep episode, as evidenced by reduced slow wave sleep and shortened rapid eye movement sleep duration during the initial sleep cycle, with later rebound of slow wave sleep during the third sleep cycle. Blocking this alerting, sleeplightening effect from light with the glasses may explain the improved sleep quality. ${ }^{35}$ Sumova et al demonstrated dual night-time melatonin peaks in patients with ADHD, which they hypothesized could mediate the sleep disruption observed in ADHD/sleep studies. ${ }^{36}$ Similarly, by reducing exposure to blue wavelength light, the glasses may normalize melatonin output and decrease sleep disruptions, thereby improving sleep quality. The relationship between circadian rhythms and sleep quality requires further study. Of interest, even though depression, anxiety, and other psychiatric comorbid conditions were exclusion criteria for this study, the subjects showed a significant decrease in anxiety following the intervention.

The results of this study confirm the findings of previous intervention studies using glasses. One study of insomnia subjects comparing the efficacy of blue wavelength lightfiltering glasses with yellow-tinted "control" glasses showed significant improvement in sleep quality and mood (as measured by sleep diary) and no change in sleep timing measures, such as time to bed or wake-time following use. ${ }^{22}$ Shift-work studies with polarized lenses also showed improvement primarily in quality rather than circadian measures. ${ }^{23}$

In reference to the feasibility of the use of these glasses in ADHD subjects, only two thirds of participating subjects were able to complete the study. Prospective subjects were invited to participate in the study and were not heavily vetted for compliance capability. Completing sleep diaries is known to be difficult for ADHD patients, ${ }^{13}$ who have trouble sustaining attention for repetitive tasks, particularly paperwork. The subjects in this study were highly variable in their degree of compliance and adhered to the protocol only to a moderate degree, maintaining a moderate lighting environment for a little more than half the time recommended and wearing the glasses for, on average, 2.5 hours per night, which was less than the recommended minimum of 3 hours. Supportive spouses who gave reminders improved compliance. Despite only partial compliance with the protocol instructions, improvement in insomnia/sleep quality symptoms was noted, suggesting at least some therapeutic benefit from the intervention. Regardless, instruction must be carefully given and capacity for compliance assessed before a patient is encouraged to buy the glasses. A spouse should be involved in treatment if at all possible. Use of validated scales before and after treatment would assist in more objective determination of benefit. ADHD subjects who are able to comply more fully with instructions would be the best candidates for treatment with these glasses and might be expected to have the most improvement in their circadian delay. Future study designs for subjects with ADHD insomnia should take compliance difficulties into account, and the high prevalence and morbidity of the condition warrants continued research, however arduous, into efficacious treatments.

A potential limitation of this study is the wide age range of the subjects, and further studies of younger versus older subjects are needed. All data gathered in this study were self-reported. To avoid the confounding effect of insufficient dark-time to wear the glasses, we terminated the study before the days became significantly longer, limiting the sample size. Confirmation of these findings will require larger groups, including the different insomnia subtypes (delayed circadian rhythm sleep disorder versus nondelayed ADHD insomnia) and objective assessments of sleep quality, melatonin levels, and other circadian measures to determine if this and other chronobiological interventions have utility. Although a strong placebo effect is observed in insomnia studies, a placebo effect was less likely in the present study because the participants were expecting an alteration in sleep timing, which was not improved by the intervention overall. However, sleep quality was noted to improve in all cases, even in subjects who reported lack of any type of improvement in sleep.

\section{Conclusion}

Despite moderate compliance with intervention instructions, subjects who completed the intervention using blue lightblocking glasses showed subjectively improved sleep quality on a number of measures and reduced anxiety. Subjects with an initial delayed midsleep time after 4:15 am showed a trend towards earlier sleep following treatment. Blue wavelength light-blocking glasses are a viable treatment for subjects with ADHD insomnia who can comply with their use, especially those subjects with greater sleep delays. Larger more objective studies of subjects who are mostly delayed might reveal significant advances in circadian rhythm in addition to furthering our understanding of how evening light exposure affects sleep quality. 


\section{Acknowledgment}

The authors thank LowBlueLights.com of Photonic Developments LLC and the Lighting Innovations Institute for donating the glasses used in this study.

\section{Disclosure}

The authors report no conflicts of interest in this work.

\section{References}

1. Kessler RC, Adler L, Barkley R, et al. The prevalence and correlates of adult ADHD in the United States: results from the National Comorbidity Survey Replication. Am J Psychiatry. 2006;163:716-723.

2. Philipsen A, Hornyak M, Riemann D. Sleep and sleep disorders in adults with attention deficit/hyperactivity disorder. Sleep Med Rev. 2006;10: 399-405.

3. Van Veen MM, Kooij JJ, Boonstra AM, Gordijn MC, Van Someren EJ. Delayed circadian rhythm in adults with attention-deficit/hyperactivity disorder and chronic sleep-onset insomnia. Biol Psychiatry. 2010;67: 1091-1096.

4. Fargason RE, Gamble KL, Avis K, Cates M, May R, Besing R. Ramelteon for insomnia related to attention-deficit hyperactivity disorder. Psychopharmacol Bull. 2011;44:1-22.

5. Fargason RE, White S, Hollar A, Gamble K. Adults with attentiondeficit hyperactivity disorder without insomnia have subclinical sleep disturbance but not circadian delay: an ADHD phenotype? J Atten Disord. September 20, 2012. [Epub ahead of print.]

6. Kissling C, Retz W, Wiemann S, et al. A polymorphism at the $3^{\prime}$-untranslated region of the CLOCK gene is associated with adult attention-deficit hyperactivity disorder. Am J Med Genet B Neuropsychiatr Genet. 2008;147:333-338.

7. Xu X, Breen G, Chen CK, Huang YS, Wu YY, Asherson P. Association study between a polymorphism at the 3 '-untranslated region of CLOCK gene and attention deficit hyperactivity disorder. Behav Brain Funct. 2010;6:48.

8. Schmidt TM, Chen SK, Hattar S. Intrinsically photosensitive retinal ganglion cells: many subtypes, diverse functions. Trends Neurosci. 2011;34:572-580.

9. Van der Heijden KB, Smits MG, Van Someren EJ, Gunning WB. Idiopathic chronic sleep onset insomnia in attention-deficit/ hyperactivity disorder: a circadian rhythm sleep disorder. Chronobiol Int. 2005;22:559-570.

10. Ingeborg M, van Geijlswijk IM, Korzilius HP, Smits MG. The use of exogenous melatonin in delayed sleep phase disorder: a meta-analysis. Sleep. 2010;33:1605-1614.

11. Burgess HJ, Revell VL, Molina TA, Eastman CI. Human phase response curves to three days of daily melatonin: $0.5 \mathrm{mg}$ versus $3.0 \mathrm{mg}$. J Clin Endocrinol Metab. 2010;95:3325-3331.

12. Burgess HJ, Revell VL, Eastman CI. A three pulse phase response curve to three milligrams of melatonin in humans. J Physiol. 2008;586: 639-647.

13. Rybak YE, McNeely HE, Mackenzie BE, Jain UR, Levitan RD. An open trial of light therapy in adult attention-deficit/hyperactivity disorder. J Clin Psychiatry. 2006;67:1527-1535.

14. Terman M. Evolving applications of light therapy. Sleep Med Rev. 2007;11:497-507.

15. Benedetti F, Dallaspezia S, Fulgosi MC, Barbini B, Colombo C, Smeraldi E. Phase advance is an actimetric correlate of antidepressant response to sleep deprivation and light therapy in bipolar depression. Chronobiol Int. 2007;24:921-937.

16. Gooley JJ, Chamberlain K, Smith KA, et al. Exposure to room light before bedtime suppresses melatonin onset and shortens melatonin duration in humans. J Clin Endocrinol Metab. 2011;96:E463-E472.
17. Gooley JJ, Rajaratnam SM, Brainard GC, Kronauer RE, Czeisler CA Lockley SW. Spectral responses of the human circadian system depend on the irradiance and duration of exposure to light. Sci Transl Med. 2010;2:31-33.

18. Johnson JE. Insomnia, alcohol, and over-the-counter drug use in older urban women. J Community Health Nurs. 1997;14:181-188.

19. Rosenberg RP. Sleep maintenance insomnia: strengths and weaknesses of current pharmacologic therapies. Ann Clin Psychiatry. 2006;18: $49-56$.

20. Kayumov L, Casper RF, Hawa RJ, et al. Blocking low-wavelength light prevents nocturnal melatonin suppression with no adverse effect on performance during simulated shift work. J Clin Endocrinol Metab. 2005;90:2755-2761.

21. Sasseville A, Paquet N, Sevigny J, Hebert M. Blue blocker glasses impede the capacity of bright light to suppress melatonin production. J Pineal Res. 2006;41:73-78.

22. Burkhart K, Phelps JR. Amber lenses to block blue light and improve sleep: a randomized trial. Chronobiol Int. 2009;26:1602-1612.

23. Sasseville A, Benhaberou-Brun D, Fontaine C, Charon MC, Hebert M. Wearing blue-blockers in the morning could improve sleep of workers on a permanent night schedule: a pilot study. Chronobiol Int. 2009;26:913-925.

24. Wirz-Justice A, Bromundt V, Cajochen C. Circadian disruption and psychiatric disorders: the importance of entrainment. Sleep Med Clin. 2009;4:273-284.

25. Phelps J. Dark therapy for bipolar disorder using amber lenses for blue light blockade. Med Hypotheses. 2008;70:224-229.

26. Sletten TL, Revell VL, Middleton B, Lederle KA, Skene DJ. Age-related changes in acute and phase-advancing resonses to monochromatic light. J Biol Rhythms. 2009;24:73-89.

27. Friedman L, Zeitzer JM, Kushida C, et al. Scheduled bright light for treatment of insomia in older adults. $J$ Am Geriatr Soc. 2009;57:441-452.

28. Harada T. Effects of evening light conditions on salivary melatonin of Japanese junior high school students. J Circadian Rhythms. 2004;2:4.

29. Buysse DJ, Reynolds CF III, Monk TH, Berman SR, Kupfer DJ. The Pittsburgh Sleep Quality Index: a new instrument for psychiatric practice and research. Psychiatry Res. 1989;28:193-213.

30. Backhaus J, Junghanns K, Broocks A, Riemann D, Hohagen F. Testretest reliability and validity of the Pittsburgh Sleep Quality Index in primary insomnia. J Psychosom Res. 2002;53:737-740.

31. Buysse DJ, Hall ML, Strollo PJ, et al. Relationships between the Pittsburgh sleep quality index (PSQI), Epworth sleepiness scale (ESS), and clinical/polysomnographic measures in a community sample. J Clin Sleep Med. 2008;4:563-571.

32. Zavada A, Gordijn MC, Beersma DG, Daan S, Roenneberg T. Comparison of the Munich chronotype questionnaire with the Horne-Ostberg's morningness-eveningness score. Chronobiol Int. 2005;22:267-278

33. Scharf MB, Roth T, Vogel GW, Walsh JK. A multicenter, placebo-controlled study evaluating zolpidem in the treatment of chronic insomnia. J Clin Psychiatry. 1994;55:192-199.

34. Zammit GK, McNabb LJ, Caron J, Amato DA, Roth T. Efficacy and safety of eszopiclone across 6-weeks of treatment for primary insomnia. Curr Med Res Opin. 2004;20:1979-1991.

35. Munch M, Kobialka S, Steiner R, Oelhafen P, Wirz-Justice A, Cajochen C. Wavelength-dependent effects of evening light exposure on sleep architecture and sleep EEG power density in men. Am J Physiol Regul Integr Comp Physiol. 2006;290:R1421-R1428.

36. Novakova M, Paclt I, Ptacek R, Kuzelova H, Hajek I, Sumova A. Salivary melatonin rhythm as a marker of the circadian system in healthy children and those with attention-deficit/hyperactivity disorder Chronobiol Int. 2011;28:630-637. 


\section{Publish your work in this journal}

ChronoPhysiology and Therapy is an international, peer-reviewed, open access journal focusing on research into the cyclic variations and rhythmicity in physiological processes in the body and the research and development and optimal timing of administration of therapeutic targets to achieve improved outcomes and quality of life for the patient. The

manuscript management system is completely online and includes a very quick and fair peer-review system. Visit http://www.dovepress.com/ testimonials.php to read real quotes from published authors.

Submit your manuscript here: http://www.dovepress.com/chronophysiology-and-therapy-journal 